\title{
Intra-operative blood transfusion significantly increases the risk of post-operative pulmonary embolism
}

\author{
Qiaojun Zeng $^{1 \#}$, Shufang Tan ${ }^{2 \#}$, Qiang Bao ${ }^{1}$, Shanping Jiang ${ }^{1}$ \\ ${ }^{1}$ Department of Pulmonary and Critical Care Medicine, ${ }^{2}$ Department of Operating Room, Sun Yat-Sen Memorial Hospital, Sun Yat-Sen University, \\ Guangzhou 510120, China \\ Contributions: (I) Conception and design: All authors; (II) Administrative support: S Tan, S Jiang; (III) Provision of study materials or patients: Q \\ Zeng, S Tan, Q Bao; (IV) Collection and assembly of data: Q Zeng, S Tan, Q Bao; (V) Data analysis and interpretation: Q Zeng, S Tan, Q Bao; (VI) \\ Manuscript writing: All authors; (VII) Final approval of manuscript: All authors. \\ \#These authors are the co-first authors. \\ Correspondence to: Shanping Jiang. Department of Pulmonary and Critical Care Medicine, Sun Yat-Sen Memorial Hospital, Sun Yat-Sen University, \\ Guangzhou 510120, China. Email: jiangshp@mail.sysu.edu.cn.
}

Background: Pulmonary embolism (PE) is a serious and preventable complication after surgery. Blood transfusion is a common event during surgery. The aim of the present study was to identify whether intraoperative blood product transfusions increase the risk of symptomatic post-operative PE.

Methods: A retrospective, single-center case-control study at Sun Yat-Sen Memorial Hospital, Sun YatSen University between 1 January 2013 and 31 December 2016 was performed. Adult hospitalized patients with confirmed symptomatic PE after surgery were defined as case group. Each patient was matched with two adult patients without symptomatic PE who underwent the same procedure on the same day or within one week. Perioperative data especially detailed blood transfusion was collected. Forward stepwise logistic regression analysis was performed.

Results: During the study period, altogether 188,512 procedures were included, and postoperative PE were confirmed in $40(0.02 \%)$ patients. Twelve (30.0\%) case patients and $16(20.0 \%)$ control patients received intra-operative blood transfusion. Intra-operative blood transfusion was revealed as a significant predictor for symptomatic post-operative PE (OR 80.669, 95\% CI: 3.312-1,964.641, P=0.007), along with female sex (OR 86.921, 95\% CI: 4.242-1,781.124, P=0.004), older age (OR 1.078, 95\% CI: 1.005-1.156, P=0.035), longer length of stay before surgery (OR 1.124, 95\% CI: 1.040-1.214, P=0.003) and longer operation time (OR $1.013,95 \%$ CI: $1.002-1.025, \mathrm{P}=0.024)$.

Conclusions: Intra-operative blood transfusion was associated with an increased risk of symptomatic postoperative PE. PE prophylaxis and selective screening should be considered in patients who had received intra-operative blood transfusion, especially in female patients.

Keywords: Pulmonary embolism (PE); blood transfusion; risk factors; surgery

Submitted Oct 29, 2018. Accepted for publication Oct 10, 2019.

doi: $10.21037 /$ jtd.2019.11.10

View this article at: http://dx.doi.org/10.21037/jtd.2019.11.10

\section{Introduction}

Pulmonary embolism (PE) is the most common preventable cause of hospital death and the number-one strategy to improve patient safety in hospitals $(1,2)$. It is well known that there is a high risk of PE after surgery $(1,2)$. The incidence of perioperative $\mathrm{PE}$ varied by type of surgical procedure, $1.6 \%$ for general surgery, $1.5-2 \%$ for thoracic surgery, $0.7-30 \%$ for all orthopedic surgical procedures $(3,4)$. In a postmortem study, mortality associated with postoperative $\mathrm{PE}$ is as high as $28.9 \%$ (5). Various factors for venous thromboembolism (VTE) have been well 
established, including female sex, older age, active neoplasia, history of previous deep venous thrombosis (DVT) or $\mathrm{PE}$, trauma, immobilization and infection (6). There is a large amount of clinical data documenting the safety and efficacy of routine thromboprophylaxis in patients undergoing surgery $(1,7)$. However, bleeding complications cannot be ignored $(8,9)$. Moreover, even with aggressive VTE prophylaxis, this event sill occurs and suggests other undefined factors may play a role in VTE occurrence (10).

Blood transfusion is a common event in patients undergoing surgery in an attempt to correct anemia and coagulation parameters during or after the operations. But Yeh et al. found that postoperative transfusion was associated with a worse outcome in pancreatic cancer patients with pancreaticoduodenectomy (11). Moreover, blood transfusion is demonstrated to be associated with an increased risk of VTE in cancer patients, due to its proinflammatory and immunomodulatory nature $(12,13)$. However, the effect of intra-operative blood transfusion and $\mathrm{PE}$ is less frequently described. So, the aim of our study was to evaluate whether intra-operative banked allogenic blood product transfusions increase the risk of symptomatic postoperative PE.

\section{Methods}

\section{Patient population}

We performed a retrospective study at Sun Yat-Sen Memorial Hospital, Sun Yat-Sen University between 1 January 2013 and 31 December 2016. Case subjects were in-hospital patients, aged over 18 years, with an objectively documented symptomatic PE after a surgical operation. The diagnostic criteria for PE were either: (I) a positive helical computed tomography with a central filling defect outlined by contrast material or a complete occlusion in a segmental or more proximal pulmonary artery; or (II) a pulmonary angiography with an intra luminal filling defect or a sharp cut-off in a vessel at least $2 \mathrm{~mm}$ in diameter. Patients who had previously diagnosed as $\mathrm{PE}$ or preoperatively diagnosed as symptomatic $\mathrm{PE}$ by helical computed tomography or pulmonary angiography were excluded. Forty clinically symptomatic and imaging-confirmed cases of PE after a surgical operation were identified. Each patient was then matched with two adult patients without symptomatic $\mathrm{PE}$ who underwent the same operation on the same day or within one week. If more than 3 patients had the same surgery on the same day, the nearest patients in the surgery opening time were chose. The same surgery is defined as having the same International Classification of Disease, $9^{\text {th }}$ Revision, Clinical Modification (ICD-9-CM) code. Although considered, age and sex were not included in the matching process. Matching on these parameters would prevent their use in the risk factor analysis.

\section{Study design}

A retrospective observational cohort study was used. We reviewed the medical records of all the patients in detail. Detailed blood transfusion data were collected on all transfused patients including product type and amount. Others including demographic, information on comorbid diseases, medications, surgical procedure, operative time, type of anesthesia, electrocautery and 30-day post-operation mortality during surgery were also recorded.

\section{Statistical analysis}

Continuous variables were described as means \pm SD. Means were compared using Student's $t$-test. Medians and comparative analysis were executed with MannWhitney U test, Kruskal-Wallis test, or chi-square test when appropriate. Potential risk factors for perioperative thromboembolic events were assessed using conditional logistic regression, making use of the 1:2 matched-pair study design. All potential risk factors were evaluated by forward stepwise logistic regression analysis in a multivariate model ( $\mathrm{P}$ for entry $<0.05$, for exit $>0.10$ ) used to identify perioperative predictors of $\mathrm{PE}$. The results were reported as odds ratios (ORs) and 95\% confidence intervals (95\% CIs). All $\mathrm{P}$ values are 2-tailed and statistically significance was set at $\mathrm{P}<0.05$. All statistical analyses were performed using the SPSS version 23.0.

\section{Results}

Between 1 January 2013 and 31 December 2016, there were 188,512 surgical operations, of which 40 patients (13 males and 27 females) satisfied criteria for postoperative $\mathrm{PE}$, resulting in an incidence of $0.02 \%$. Pulmonary emboli were recognized at 3.5 days (median; range, 1 to 46 days) after surgery. Almost all of the patients were documented by helical computed tomography, except one by pulmonary angiography. Of the 40 cases, 10 patients underwent abdomen surgery, 7 underwent gynecological surgery, 6 underwent orthopedic surgery, 6 underwent cardiovascular 
Table 1 Baseline characteristics in case and control patients.

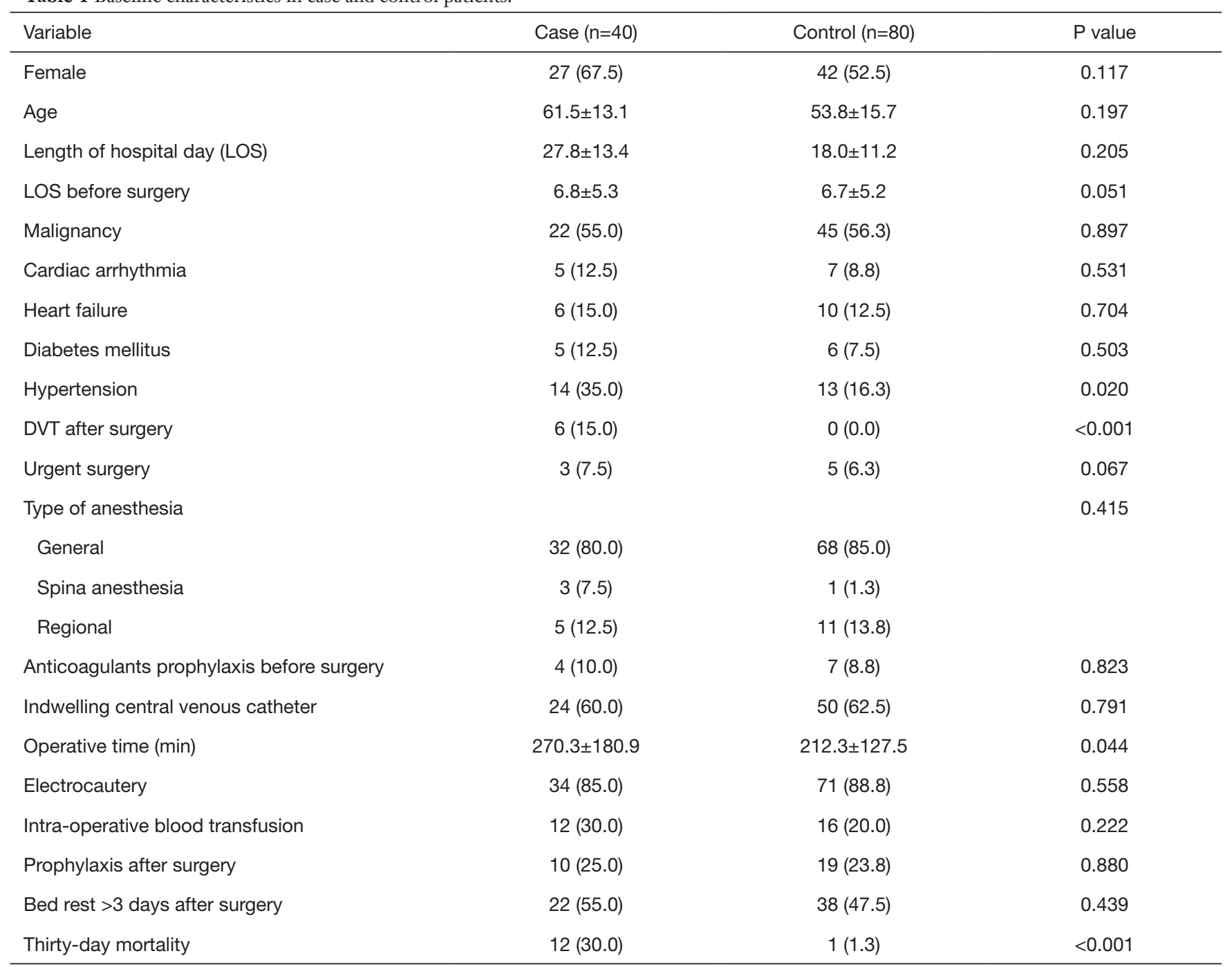

Values are $\mathrm{n}(\%)$ unless otherwise noted. LOS, length of stay; DVT, deep vein thrombosis.

surgery, 4 underwent breast surgery, 3 underwent thoracic surgery, 2 underwent cranial neurosurgery and 2 underwent urological surgery. Three patients in the case group underwent urgent surgery, while 5 patients underwent urgent surgery in the control group.

Comparison between 40 patients with symptomatic $\mathrm{PE}$ and 80 patients without symptomatic PE were summarized in Table 1. All patients did not have signs/symptoms of DVT before surgery. There were no differences in sex, age and intra-operative blood transfusion between the case patients and control patients. Twelve (30.0\%) case patients received intra-operative blood transfusion. All of them received at least 2-unit transfusion of leukocyte-reduced red blood cell (RBC). Three patients transfused 1-unit platelet, at least $200 \mathrm{~mL}$ fresh frozen plasma (FFP) and RBC. Five patients transfused both FFP and RBC. Seven (58.3\%) of the 12 case patients who received intra-operative blood transfusion were died. Among control patients, 16 (20.0\%) received intra-operative blood transfusion. Four patients only received at least 1-unit leukocyte-reduced RBC and 1 patient only received $400 \mathrm{~mL}$ FFP transfusion. Two patients transfused 1-unit platelet, at least $200 \mathrm{~mL} \mathrm{FFP} \mathrm{and}$ at least 2-unit RBC. Nine patients transfused both FFP and RBC. One $(6.3 \%)$ of the 16 control patients who received intra-operative blood transfusion was died. Hypertension and DVT after surgery were more frequent in case patients 
Table 2 Forward stepwise logistic regression analysis of potential risk factors for symptomatic post-operative pulmonary embolism

\begin{tabular}{lccc}
\hline Variable & OR & $95 \% \mathrm{Cl}$ & $\mathrm{P}$ value \\
\hline Female & 86.921 & $4.242-1,781.124$ & 0.004 \\
Age & 1.078 & $1.005-1.156$ & 0.035 \\
LOS before surgery & 1.124 & $1.040-1.214$ & 0.003 \\
Operative time (min) & 1.013 & $1.002-1.025$ & 0.024 \\
Intra-operative blood transfusion & 80.669 & $3.312-1,964.641$ & 0.007 \\
\hline
\end{tabular}

LOS, length of stay; OR, odds ratios; $\mathrm{Cl}$, confidence intervals.

than in control patients $(35.0 \%$ versus $16.3 \%, \mathrm{P}=0.020$; $15.0 \%$ versus $0.0 \%, \mathrm{P}<0.001$, respectively). In addition, Case patients had longer operating time and higher 30-day mortality $(270.3 \pm 180.9$ versus $212.3 \pm 127.5, \mathrm{P}=0.044 ; 30.0 \%$ versus $1.3 \%, \mathrm{P}<0.001$, respectively).

By forward stepwise logistic regression analysis, intraoperative blood transfusion was demonstrated to be a significant predictor for PE after surgery (OR 80.669, 95\% CI: 3.312-1,964.641, $\mathrm{P}=0.007$ ) (Table 2). Other independent risk factors for post-operative $\mathrm{PE}$ were female sex (OR 86.921, 95\% CI: 4.242-1,781.124, $\mathrm{P}=0.004)$, older age (OR $1.078,95 \%$ CI: $1.005-1.156, \mathrm{P}=0.035)$, longer length of stay before surgery (OR 1.124, 95\% CI: 1.040-1.214, $\mathrm{P}=0.003$ ) and longer operation time (OR 1.013, 95\% CI: 1.002-1.025, $\mathrm{P}=0.024)$.

\section{Discussion}

In this study, we reviewed cases of symptomatic PE after 188,512 consecutive surgeries at a single institution and found a low rate of symptomatic post-operative PE $(0.02 \%)$. The median time between surgery and the diagnosis of PE was 3.5 days. This was consistent with other studies suggesting that PE mainly occurs during the first postoperative week $(9,14,15)$.

Blood product transfusions are frequently utilized during surgery. Intra-operative blood transfusion was demonstrated to be a significant risk factor for the development of $\mathrm{PE}$ in our study. The relationship between blood transfusion and development of VTE has been previously reported (16-20). Rogers et al. found that transfusion of $>4 \mathrm{U}$ packed red blood cell (PRBC) preoperatively was independently associated with the development of VTE (16). Xenos et al. reported that intra-operative $\mathrm{PRBC}$ blood transfusion was a significant risk factor for the development of VTE and the risk increased with increasing number of units transfused (17). Among 1,070 patients undergoing cardiac surgery, intra- or post-operative PRBC transfusion is associated with increased risk of DVT in a dose-dependent fashion that is exacerbated when accompanied with FFP (18). A study of women with stage IIIC-V adnexal or peritoneal cancer, postoperative FFP transfusion was associated with increased risk of DVT and PE (19). Moreover, Nilsson et al. found that perioperative allogeneic blood transfusion was associated with an increase of developing VTE in women, but not in men (20). In our study, female sex was also a significant risk factor for the development of PE. Thus, the surgeon maybe should weigh the risk and benefits of blood product transfusions in the perioperative period, especially in female patients.

There are many possible mechanisms that might explain for this phenomenon. Transfused blood, especially RBC blood transfusion can disrupt the balance of coagulation factors and modulates the inflammatory cascade (17). Storage of red cells can be associated with an increase red cell aggregability and abnormal morphology which might contribute to blood flow disorders (21). Normal mature RBCs do not interact with endothelium, but they become highly sticky when they have been stored longer, and this adhesion of vascular endothelium can contribute substantially to microvascular occlusions associated with thrombosis (22). With prolonged storage, oxidative stress increases and results in lipid peroxidation, protein oxidation and reduced integrity of the erythrocyte membrane-all contributing to the formation of extra-cellular microvesicles or 'micro-particles (MP)' and hemolysis (23). MPs are $0.1-1.0 \mu \mathrm{m}$ in size and are released in blood flow by various types of cells (24). Hemolysis release free hemoglobin and heme that are toxic to many cells and tissues (22). Both MPs and hemolysis were associated with a reduction in vasodilation, platelet adhesion and aggregation, and exacerbation of inflammation $(12,23)$. In addition, 
platelet MP might work synergistically with red-cell derived MP in mediating some transfusion-related thrombotic complications (25). The addition of plasma may also affect the coagulation balance by adding pro-coagulants. Nitric oxide (NO) also has a fundamental role in vascular health by regulating vasodilation, compromising blood flow, inhibiting both platelet aggregation and endothelial adhesion molecule expression (23). Reynolds et al. found that banked blood is severely depleted in NO which may lead to augment thrombosis, microcirculatory perturbations or injury in susceptible hosts $(23,26)$. Moreover, massive intra-operative blood transfusion might mean more blood loss, leading to increased use of hemostatic drugs and further deterioration of coagulation and thrombosis.

Bed rest $>3$ days after surgery in our study was not associated with enhanced risk of $\mathrm{PE}$ development. However, longer length of stay before surgery was associated with increased risk of postoperative PE. Gillinov et al. also found prolonged preoperative bed rest as a risk factor for $\mathrm{PE}$ in the cardiac surgical patient (27). Greaves et al. analyzed 242,670 patients undergoing colorectal surgery and found that preoperative hospitalization was an independent risk factor for VTE and each day of preoperative hospitalization increased the odds of VTE (28). The longer preoperative period might contribute to the onset of $\mathrm{PE}$ by reducing mobility and causing venous stasis $(28,29)$.

\section{Limitations}

This study had several limitations. First, this study was a case-control review with a relatively small sample size, which might influence its generalizability. Nonetheless, the effect size was strong enough that a statistically significant impact of these variables could be detected. Second, the lack of a strictly matched design between the two groups made it difficult to balance all confounding factors. Third, length of storage was not available. Therefore, we cannot comment on the effect of transfusion of older units on development of post-operative $\mathrm{PE}$.

\section{Conclusions}

In this study, symptomatic PE occurred infrequently after surgery $(0.02 \%)$. Intra-operative blood transfusion and female sex were associated with increased risk of symptomatic post-operative PE. PE prophylaxis and selective screening should be considered in patients who had received intra-operative blood transfusion, especially in female patients.

\section{Acknowledgments}

Funding: This work was supported by the National Science Foundation of China (81670022); Science Foundation of Guangdong Province (2017A030313681); and Funding from Guangzhou Science and Technology Bureau (201704020123).

\section{Footnote}

Conflicts of Interest: The authors have no conflicts of interest to declare.

Ethical Statement: The authors are accountable for all aspects of the work in ensuring that questions related to the accuracy or integrity of any part of the work are appropriately investigated and resolved. All procedures performed in our study involving human participants were in accordance with the ethical standards of the institutional and national research committee (SYSEC-KYKS-2018-049), and with the 1964 Helsinki declaration and its later amendments or comparable ethical standards. For our study on patient data retrieved from hospital medical record system, an informed consent form is not required. The patient's personal data have been secured.

\section{References}

1. Geerts WH, Bergqvist D, Pineo GF, et al. Prevention of venous thromboembolism: American College of Chest Physicians Evidence-Based Clinical Practice Guidelines (8th Edition). Chest 2008;133:381S-453S.

2. Geerts WH, Pineo GF, Heit JA, et al. Prevention of Venous Thromboembolism. Chest 2004;126:338S-400S.

3. Mantilla CB, Horlocker TT, Schroeder DR, et al. Frequency of myocardial infarction, pulmonary embolism, deep venous thrombosis, and death following primary hip or knee arthroplasty. Anesthesiology 2002;96:1140-6.

4. Desciak MC, Martin DE. Perioperative pulmonary embolism: diagnosis and anesthetic management. J Clin Anesth 2011;23:153-65.

5. Lindblad B, Eriksson A, Bergqvist D. Autopsy-verified pulmonary embolism in a surgical department: analysis of the period from 1951 to 1988. Br J Surg 1991;78:849-52.

6. Konstantinides SV, Torbicki A, Agnelli G, et al. 2014 ESC guidelines on the diagnosis and management of 
acute pulmonary embolism. Eur Heart J 2014;35:3033-69, 69a-69k.

7. Working Group for Audit about Perioperative Accident and Pulmonary Embolism of Japanese Society of Anesthesiologists. Perioperative risk factors for death among patients with symptomatic pulmonary thromboembolism. J Anesth 2017;31:478-82.

8. Hamilton MG, Yee WH, Hull RD, et al. Venous thromboembolism prophylaxis in patients undergoing cranial neurosurgery: a systematic review and metaanalysis. Neurosurgery 2011;68:571-81.

9. Gonzalez Della Valle A, Lee YY, Saboeiro G, et al. OneYear All-Cause Mortality of Patients Diagnosed as Having In-Hospital Pulmonary Embolism After Modern Elective Joint Arthroplasty Is Low And Unaffected By Radiologic Severity. J Arthroplasty 2016;31:473-9.

10. Gangireddy C, Rectenwald JR, Upchurch GR, et al. Risk factors and clinical impact of postoperative symptomatic venous thromboembolism. J Vasc Surg 2007;45:335-41; discussion 41-2.

11. Yeh JJ, Gonen M, Tomlinson JS, et al. Effect of blood transfusion on outcome after pancreaticoduodenectomy for exocrine tumour of the pancreas. Br J Surg 2007;94:466-72.

12. Rogers MA, Levine DA, Blumberg N, et al. Triggers of hospitalization for venous thromboembolism. Circulation 2012;125:2092-9.

13. Khorana AA, Francis CW, Blumberg N, et al. Blood transfusions, thrombosis, and mortality in hospitalized patients with cancer. Arch Intern Med 2008;168:2377-81.

14. Krych AJ, Sousa PL, Morgan JA, et al. Incidence and Risk Factor Analysis of Symptomatic Venous Thromboembolism After Knee Arthroscopy. Arthroscopy 2015;31:2112-8.

15. Gesell MW, Gonzalez Della Valle A, Bartolome Garcia S, et al. Safety and efficacy of multimodal thromboprophylaxis following total knee arthroplasty: a comparative study of preferential aspirin vs. routine coumadin chemoprophylaxis. J Arthroplasty 2013;28:575-9.

16. Rogers SO Jr, Kilaru RK, Hosokawa P, et al. Multivariable predictors of postoperative venous thromboembolic events after general and vascular surgery: results from the patient safety in surgery study. J Am Coll Surg 2007;204:1211-21.

17. Xenos ES, Vargas HD, Davenport DL. Association of blood transfusion and venous thromboembolism after colorectal cancer resection. Thromb Res 2012;129:568-72.
18. Ghazi L, Schwann TA, Engoren MC, et al. Role of blood transfusion product type and amount in deep vein thrombosis after cardiac surgery. Thromb Res 2015;136:1204-10.

19. Abu-Rustum NR, Richard S, Wilton A, et al. Transfusion utilization during adnexal or peritoneal cancer surgery: effects on symptomatic venous thromboembolism and survival. Gynecol Oncol 2005;99:320-6.

20. Nilsson KR, Berenholtz SM, Garrett-Mayer E, et al. Association between venous thromboembolism and perioperative allogeneic transfusion. Arch Surg 2007;142:126-32; discussion 133.

21. Hovav T, Yedgar S, Manny N, et al. Alteration of red cell aggregability and shape during blood storage. Transfusion 1999;39:277-81.

22. Litvinov RI, Weisel JW. Role of red blood cells in haemostasis and thrombosis. ISBT Sci Ser 2017;12:176-83.

23. Lee JS, Gladwin MT. Bad blood: the risks of red cell storage. Nat Med 2010;16:381-2.

24. Said AS, Doctor A. Influence of red blood cell-derived microparticles upon vasoregulation. Blood Transfus 2017;15:522-34.

25. Jy W, Ricci M, Shariatmadar S, et al. Microparticles in stored red blood cells as potential mediators of transfusion complications. Transfusion 2011;51:886-93.

26. Reynolds JD, Ahearn GS, Angelo M, et al. S-nitrosohemoglobin deficiency: a mechanism for loss of physiological activity in banked blood. Proc Natl Acad Sci U S A 2007;104:17058-62.

27. Gillinov AM, Davis EA, Alberg AJ, et al. Pulmonary embolism in the cardiac surgical patient. Ann Thorac Surg 1992;53:988-91.

28. Greaves SW, Holubar SD. Preoperative Hospitalization Is Independently Associated With Increased Risk for Venous Thromboembolism in Patients Undergoing Colorectal Surgery: A National Surgical Quality Improvement Program Database Study. Dis Colon Rectum 2015;58:782-91.

29. Heatley RV, Hughes LE, Morgan A, et al. Preoperative or postoperative deep-vein thrombosis? Lancet 1976;1:437-9.

Cite this article as: Zeng Q, Tan S, Bao Q, Jiang S. Intraoperative blood transfusion significantly increases the risk of post-operative pulmonary embolism. J Thorac Dis 2019;11(12):5566-5571. doi: 10.21037/jtd.2019.11.10 